\title{
Do Pedicle Screws in Concave Apex of Scoliosis Offer Any Advantages?
}

\author{
Yogesh Kishorkant Pithwa \\ Department of Spine Surgery, HOSMAT Hospital, Bengaluru, India
}

Study Design: Retrospective analysis of prospectively collected data.

Purpose: To assess the relative advantages of implant constructs with and without pedicle screws in the concave apex for correcting scoliosis.

Overview of Literature: Concave apical pedicles in scoliosis can be narrow and dysplastic. Neural structures also migrate toward concavity, leaving little room for error while inserting pedicle screws into the concave apex.

Methods: Patients ( $n=35$ ) undergoing scoliosis surgery from September 2004 to September 2009 with minimum 5-year follow-up period were included. Exclusion criteria were pseudarthrosis, implant failure, infection, anterior release surgery, corrective osteotomies, incomplete data, constructs not involving anchors at the apex of the curve, and kyphoscoliosis. Curves were classified into two groups as follows: group A, with screws alone anchoring the convex apex and the correction performed from the convex side and group B, with screws anchoring the concave apex with or without convex apex purchase and the correction performed from the concave side. Results: Twenty-two of 35 patients were selected. In these patients, 29 individual curves were selected and classified into groups A $(n=15)$ and $B(n=14)$. Both groups were comparable in terms of age, sex, and etiology (idiopathic and nonidiopathic). However, group A had larger $\left(68.53^{\circ} \pm 26.29^{\circ}\right)$ and more rigid $\left(29.04 \% \pm 18.22 \%\right.$ flexibility) curves than group $B\left(50.14^{\circ} \pm 16.89^{\circ}\right.$ with $49.87 \% \pm 25.01 \%$ flexibility) (two-tailed $p<0.05$ ). Despite this, the immediate postoperative correction was comparable between the two groups ( $\mathrm{A}$, $57.98 \% \pm 16.28 \%$; $B, 62.76 \% \pm 13.13 \%$; two-tailed $p=0.39$ ). Interestingly, group $A$ showed significantly better results in terms of the gain of instrumented correction over and above preoperative flexibility $(A, 28.94 \% \pm 8.51 \% ; B, 12.89 \% \pm 23.06 \%$; two-tailed $p=0.03$ ). There was no statistically significant difference in the correction percentage of sagittal profile between the two groups and in the loss of correction at follow-up or Scoliosis Research Society-22 scores.

Conclusions: Present study could not demonstrate any advantages associated with use of apical concave pedicle screws.

Keywords: Spine; Scoliosis; Apex; Correction; Pedicle screws

\section{Introduction}

All-pedicle screw constructs have been increasingly used to correct spinal deformities [1]. Although these have been demonstrated to have their advantages, concerns have also been raised regarding the safety of inserting screws into the concave apex of a scoliotic curve [2]. In view of the fact that neural structures migrate toward the concavity of a curve, the screws would be hugging the medial borders of concave apical pedicles. As a result, although a medial breach of up to $2 \mathrm{~mm}$ has been considered clinically safe in a non-deformed spine, the margin

Received Jun 25, 2017; Revised Oct 5, 2017; Accepted Oct 30, 2017

Corresponding author: Yogesh K. Pithwa

Sattvik Spine Foundation, No. 1721 (new no. 13), 41 “A” Cross, 18th Main Road, Jayanagar 4th “T” Block, Bengaluru, Karnataka 560041, India

Tel: +91-80-42068257, Fax: +91-80-41124695, E-mail: info@spine-surgeon.org 
of error is unavailable in cases where the concave apex screws are inserted in a scoliotic spine [3].

The present study was aimed at assessing the relative advantages of constructs with and without concave apical screws. Because spinal deformity corrections are performed by inserting the first rod, be it on the convex or the concave surface, we hypothesized that the extent of correction achieved with either option will be comparable.

\section{Materials and Methods}

This was a retrospective analysis of prospectively collected data of patients undergoing scoliosis surgery from September 2004 to September 2009 at a single center with a minimum follow-up of 5 years. Approval was obtained from the Institutional Review Board of HOSMAT Hospital for conducting this study (IRB approval no., 15$16 / 08)$. Patients with pseudarthrosis, implant failure, infection, anterior release surgery, corrective osteotomies beyond Schwab type 1, incomplete data, constructs not involving anchors at the apex of the curve, and kyphoscoliosis were excluded [4].

All surgeries were performed with a standard posterior approach by the freehand insertion of pedicle screws. A $5.5-\mathrm{mm}$ rod was used in all cases. Posterior fusion was performed using local bone along with bone graft substitutes. The extent of fusion was decided on the basis of the standard criteria [1].
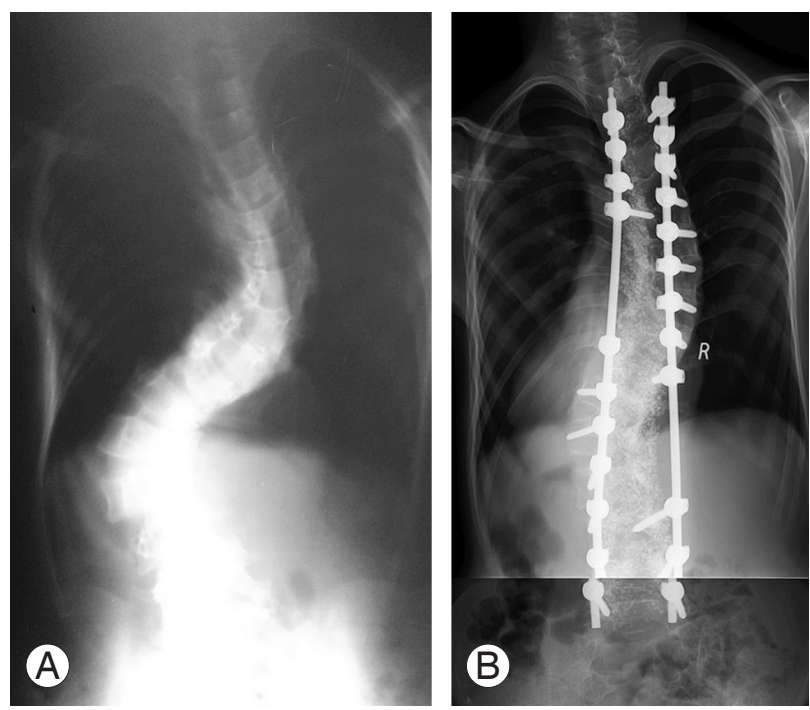

Fig. 1. Representative case of group A with pedicle screws alone anchoring the convex apex and skipping the concave apex. (A) Preoperative radiograph. (B) Postoperative radiograph.
Curves were classified into two groups as follows: group A, with screws alone anchoring the convex apex and the correction performed from the convex side and group B, with screws anchoring the concave apex with or without convex apex purchase and the correction performed from the concave side (Figs. 1, 2). In patients with double major curves, a short temporary rod was inserted from the convex side of one curve (generally the lumbar curve), and the final rod was inserted from the convex side of the other curve, extending down onto the concave side of the already-corrected first curve. The primary corrective maneuver in group A was cantilever rod correction and that in group $\mathrm{B}$ was rod rotation. Additional compression-distraction maneuvers were employed in curves $>55^{\circ}[5]$. No apical vertebral derotation maneuvers were performed.

Radiographic parameters of the magnitude of the preoperative curve, preoperative curve flexibility, percentage of immediate postoperative correction, and loss of correction at last follow-up were calculated. Most importantly, we noted the gain of instrumented correction in the immediate postoperative period over and above preoperative flexibility (Fig. 3). Thoracic kyphosis (from the superior endplate of T5 to the inferior endplate of T12) and lumbar lordosis (from the superior endplate of L1 to the superior endplate of S1) was measured during the preoperative and postoperative periods. Both groups were assessed for comparability in terms of age, sex, etiology (idiopathic and nonidiopathic), preoperative curve magnitude, and
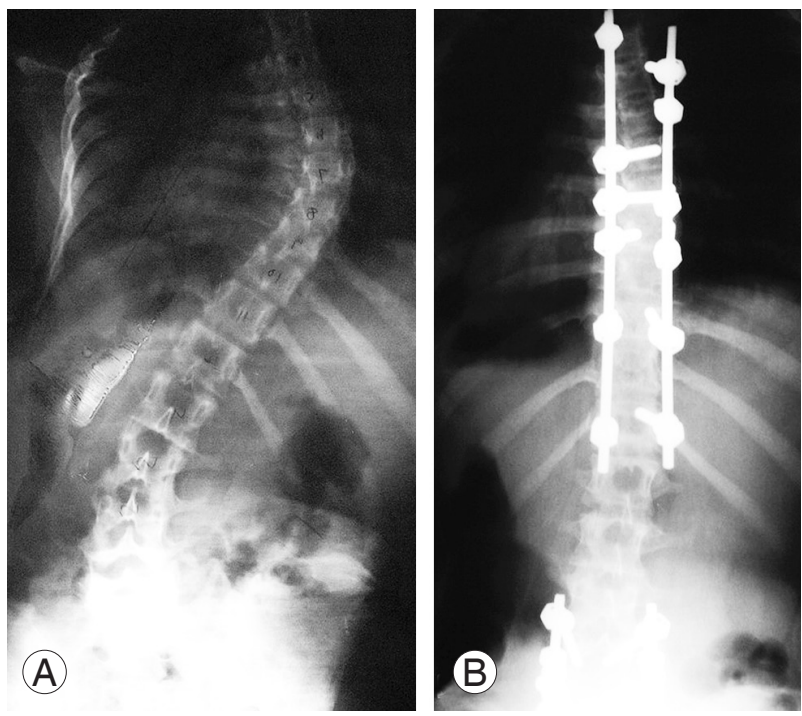

Fig. 2. Representative case of group B with pedicle screws anchoring concave apex. (A) Preoperative radiograph. (B) Postoperative radiograph. 

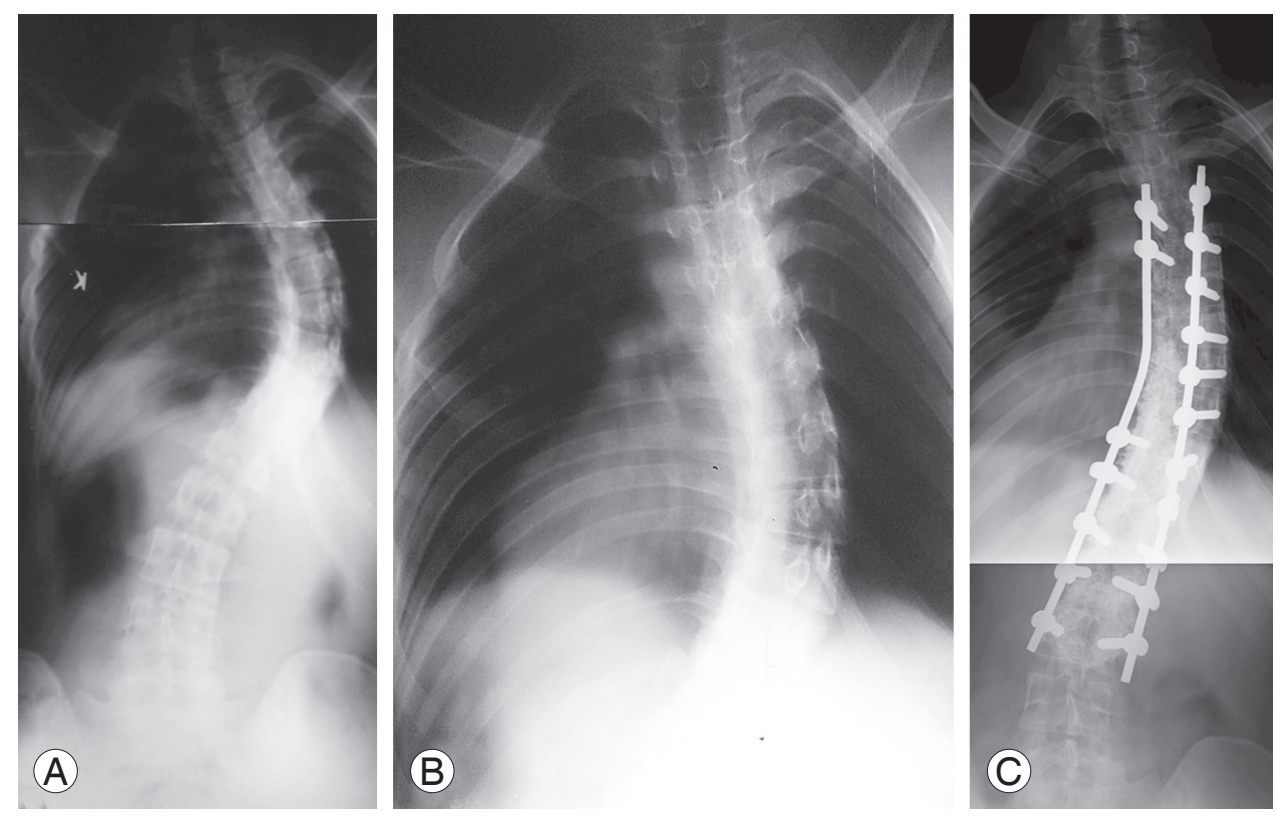

Fig. 3. Assessing the real gains of instrumentation over preoperative flexibility. (A) Preoperative standing radiograph revealing right main thoracic scoliosis of $68^{\circ}$ measured from T5 to T12. (B) Preoperative flexibility radiograph revealing the correction of this scoliosis to $46^{\circ}$. (C) Postoperative standing radiograph revealing the scoliosis of $33^{\circ}$, which is a correction of $52 \%$ over preoperative standing radiograph and $28 \%$ over the flexibility radiograph.

Table 1. Both groups were comparable in terms of age, sex, and etiology

\begin{tabular}{lccc} 
Parameter & Group A & Group B & $p$-value \\
Age (yr) & 15 (range, 11-28) & 13.5 (range, 11-25) & 0.42 \\
Sex (male:female) & $4: 7$ & $1: 7$ & 0.34 \\
Etiology (idiopathic:nonidiopathic) & $5: 10$ & $8: 6$ & 0.27 \\
\hline
\end{tabular}

Values are presented as median (range) or number. All $p$-values $>0.05$.

preoperative curve flexibility. Clinical outcomes were assessed using Scoliosis Research Society-22 (SRS-22) Questionnaire [6].

Statistical analysis was performed using statistical software, GraphPad Instat ver. 3.0 (GraphPad Software, San Diego, CA, USA). Tests used included parametric and non-parametric methods (unpaired $t$-test, Mann-Whitney $U$-test, and Fisher's exact test) where appropriate. Significance value was set at two-tailed $p<0.05$.

\section{Results}

Based on the inclusion-exclusion criteria, 22 of 35 patients were selected; seven of these patients had double major curves. Thus, 29 individual curves in these 22 patients were identified for analysis. These individual curves were classified into group A, without screws anchoring the concave apex $(\mathrm{n}=15)$, and group $\mathrm{B}$, with screws anchoring the concave apex $(\mathrm{n}=14)$.

Analysis was performed to assess the baseline comparability of the two groups. There were no statistically significant differences between the two groups in terms of age, sex, and etiology (idiopathic vis-a-vis nonidiopathic) (Table 1).

Group A had significantly larger preoperative Cobb angles than those of group B (two-tailed $p=0.03$, unpaired $t$ test with Welch correction) (Table 2). Preoperative curves in group A were also significantly less flexible than those in group B (two-tailed $p=0.02$, unpaired $t$-test with Welch correction).

Immediate postoperative correction, calculated in relation to the preoperative curve magnitude on standing 
radiographs, showed comparable correction in groups $\mathrm{A}$ and $\mathrm{B}$ (two-tailed $p=0.39$, unpaired $t$-test with Welch correction) (Table 2).

As illustrated in Fig. 3, we compared the two groups in terms of the percentage gains in correction over preoperative flexibility values. Accordingly, group A demonstrated better correction than group B (Table 2), and this difference was statistically significant (two-tailed $p=0.03$, unpaired $t$-test with Welch correction).

There were no statistically significant differences in the correction percentages of thoracic kyphosis and lumbar lordosis between the two groups (Table 3). All patients in both groups had postoperative thoracic kyphosis (T5T12) in the normal range of $10^{\circ}-40^{\circ}$.

We also assessed the difference in the number of patients with losses of $>10^{\circ}$ of scoliosis curve correction at the final follow-up [7]. At a minimum follow-up of 5 years (range, 60-72 months), only two patients, both belonging to group B, had a loss of correction of $>10^{\circ}$. This difference between the two groups was statistically insignificant (two-tailed $p=0.16$, Fisher's exact test).

As mentioned above, seven of the 22 patients selected for this study had double major curves requiring instrumentation. Of these seven patients, three patients had one curve belonging to group A and the other belonging to group B. Therefore, to compare SRS-22 scores, these three patients were excluded. This effectively led to eleven patients in group A and eight in group B. Comparison between the two groups in all total scores as well as in all individual domains revealed no statistically significant differences (Table 4). No patient in either group exhibited neurological deterioration.

\section{Discussion}

Cheng et al conducted a comparative study in patients

Table 2. Data related to preoperative and postoperative curve magnitude and related data of the two groups

\begin{tabular}{|c|c|c|c|}
\hline Parameter & Group A & Group B & $p$-value \\
\hline Preoperative Cobb angle on standing radiograph $\left({ }^{\circ}\right)$ & $68.53 \pm 26.29$ & $50.14 \pm 16.89$ & 0.03 \\
\hline Preoperative flexibility of curve (\%) & $29.04 \pm 18.22$ & $49.87 \pm 25.01$ & 0.02 \\
\hline $\begin{array}{l}\text { Immediate postoperative correction calculated in relation to preoperative } \\
\text { curve magnitude on standing radiographs }(\%)\end{array}$ & $57.98 \pm 16.28$ & $62.76 \pm 13.13$ & 0.39 \\
\hline Percentage gains in correction over preoperative flexibility values (\%) & $28.94 \pm 8.51$ & $12.89 \pm 23.06$ & 0.03 \\
\hline
\end{tabular}

Values are presented as mean \pm standard deviation.

Table 3. Percentage change in the sagittal profiles following surgery

\begin{tabular}{lccc} 
\% Change from preoperative to postoperative & Group A & Group B & $p$-value \\
Thoracic kyphosis (\%) & 0 (range, -37.5 to 46.15$)$ & 0 (range, -80.77 to 47.06$)$ & $>0.99$ \\
Lumbar lordosis $(\%)$ & 5.26 (range, -20 to 31.25$)$ & 5.44 (range, -26.15 to 104.55$)$ & 0.76 \\
\hline
\end{tabular}

Values are presented as median (range).

Table 4. Scoliosis Research Society-22 median scores

\begin{tabular}{lccc} 
Domains & Group A & Group B & $p$-value \\
Pain & 4.2 (range, 3.8-4.4) & 4.2 (range, 4-4.6) & $>0.99$ \\
Self-image & 4 (range, 3.8-4.2) & 4.2 (range, 4-4.4) & 0.1 \\
\hline Function & 4.2 (range, 3.8-4.4) & 4.2 (range, 3.8-4.4) & 0.6 \\
Mental health & 4.2 (range, 4-4.4) & $4.3($ range, 4-4.4) & 0.48 \\
Satisfaction with management & 5 (range, 4-5) & 5 (range, 4-5) & 0.48 \\
Total score & 4.32 (range, 3.92-4.48) & 4.38 (range, 3.96-4.52) & 0.26 \\
\hline
\end{tabular}

Values are presented as median (range). 
receiving one of two types of implant constructs, namely one with apical pedicle screws and the other with apical sublaminar wires [8]. Although there were no significant differences in the overall outcomes between the two groups, sublaminar wires led to higher blood loss, whereas pedicle screws led to higher implant costs. Akcali et al. [9] conducted a comparative study to assess the benefits of using concave apical purchase for correcting scoliosis. They noted better outcomes associated with the concave apical purchase. However, their study dealt with hook-constructs only. Karatoprak et al. [10] conducted a comparative study on pedicle screws and hybrid constructs. Although they noted better maintenance of correction with pedicle screws, the follow-up period for pedicle screws was shorter (29.3 months) than that for hybrid constructs (60.1 months). They also noted better apical vertebral translation with sublaminar wires than with pedicle screws. Liljenqvist et al. [11] also noted better correction with pedicle screws than with hooks. However, the two groups were not classified on the basis of concave apical purchase. Yilmaz et al. [12] studied the outcomes among three implant-construct groups, namely pedicle screws, hybrid, and hooks. The outcomes were slightly inferior in the hook group and comparable between the pedicle screw and hybrid groups. Thus, there is no unequivocal level I scientific evidence to prove or disprove the overall superiority of pedicle screw anchors on the concave apex.

The corrective force for translating the apex to midline is an additional subject of debate-whether to pull the concave side or push the convex side. Although the popular concept is to pull the concave side to the apex, pushing the convex side as the initial corrective maneuver has been promulgated by the method of vertebral coplanar alignment of spine as well as by cantilever bending $[13,14]$. In their original 2008 publication, Vallespir et al. [13] discussed the method of vertebral coplanar alignment that involved inserting tubes over screws on the convex side of scoliosis. Using one fixed and one sliding rod, they demonstrated excellent deformity correction from the convex side. A similar principle of correction from the convex side was reported by Chang [14] in 2003. Using cantilever principles, Chang [14] could demonstrate powerful correction, obviating the need for anterior release in patients with severe scoliosis with Cobb angles of $\geq 70^{\circ}$ and flexibility of $\leq 30 \%$.

The present study dealt with two groups with the pri- mary parameter of differentiation being the anchorage of the concave apex with pedicle screws to elucidate the relative advantages and disadvantages over other implant constructs. The two groups were comparable in terms of age, sex, and etiology. Group B had the anchorage of the concave apex with pedicle screws and exhibited relatively smaller preoperative curves with greater flexibility than group $\mathrm{A}$, in which patients did not have the anchorage of the concave apex with pedicle screws. Despite this, the percentage of immediate postoperative correction was comparable between the two groups. However, this assessment does not elucidate the exact gains of correction achieved by instrumentation because it compares the immediate postoperative and the preoperative standing Cobb angles. The true gains of instrumentation can only be elucidated by comparing immediate postoperative correction with the preoperative bending or traction views. Performing this analysis showed better correction in group A than in group B. The likely explanation for this improved correction in group A is that group A had cantilever correction as the main corrective force, whereas group B had rod rotation as the main corrective force. As demonstrated by Chang [14], cantilever correction seems more powerful [14]. Using a corrective maneuver from the convex side has also been associated with concerns related to decreasing thoracic kyphosis. However, postoperative sagittal profiles were comparable between the two groups in this study. Studies by Vallespir et al. [13] and Chang [14], dealing with correction from the convex side, have not shown any adverse effect on thoracic kyphosis attributable to this approach $[13,14]$.

Thoracic torsion could not be examined in this study. Although radiographic parameters of apical rib spread difference and apical rib hump prominence can be utilized for this purpose, these were not viable in the present study because a fairly large number of patients underwent thoracoplasty [15]. In addition, we could not use apical vertebral body-rib ratio because several radiographs were cropped and did not include the entire chest wall for assessment [15]. The relatively small sample size and single surgeon-single center study are additional drawbacks of this study. Although these drawbacks preclude us from reaching any dogmatic conclusion as a result of this study, these points can become the bases for future studies. 


\section{Conclusions}

In conclusion, the present study could not demonstrate any gain associated with the use of apical concave pedicle screws. A larger, prospective, multicenter study may shed more light on the risk-benefit ratio.

\section{Conflict of Interest}

No potential conflict of interest relevant to this article was reported.

\section{ORCID}

Yogesh Kishorkant Pithwa: https://orcid.org/0000-00024999-8524

\section{References}

1. Lenke LG, Kuklo TR, Ondra S, Polly DW Jr. Rationale behind the current state-of-the-art treatment of scoliosis (in the pedicle screw era). Spine (Phila Pa 1976) 2008;33:1051-4.

2. Sarwahi V, Sugarman EP, Wollowick AL, Amaral TD, Lo Y, Thornhill B. Prevalence, distribution, and surgical relevance of abnormal pedicles in spines with adolescent idiopathic scoliosis vs. no deformity: a CT-based study. J Bone Joint Surg Am 2014;96:e92.

3. Modi HN, Suh SW, Fernandez H, Yang JH, Song HR. Accuracy and safety of pedicle screw placement in neuromuscular scoliosis with free-hand technique. Eur Spine J 2008;17:1686-96.

4. Schwab F, Blondel B, Chay E, et al. The comprehensive anatomical spinal osteotomy classification. Neurosurgery 2015;76 Suppl 1:S33-41.

5. Panjabi MM, Brand RA Jr, White AA 3rd. Threedimensional flexibility and stiffness properties of the human thoracic spine. J Biomech 1976;9:185-92.

6. Asher M, Min Lai S, Burton D, Manna B. The reliability and concurrent validity of the scoliosis re- search society-22 patient questionnaire for idiopathic scoliosis. Spine (Phila Pa 1976) 2003;28:63-9.

7. Betz RR, Harms J, Clements DH 3rd, et al. Comparison of anterior and posterior instrumentation for correction of adolescent thoracic idiopathic scoliosis. Spine (Phila Pa 1976) 1999;24:225-39.

8. Cheng I, Kim Y, Gupta MC, et al. Apical sublaminar wires versus pedicle screws: which provides better results for surgical correction of adolescent idiopathic scoliosis? Spine (Phila Pa 1976) 2005;30:2104-12.

9. Akcali O, Alici E, Kosay C. Apical instrumentation alters the rotational correction in adolescent idiopathic scoliosis. Eur Spine J 2003;12:124-9.

10. Karatoprak O, Unay K, Tezer M, Ozturk C, Aydogan $\mathrm{M}$, Mirzanli C. Comparative analysis of pedicle screw versus hybrid instrumentation in adolescent idiopathic scoliosis surgery. Int Orthop 2008;32:523-8.

11. Liljenqvist U, Lepsien U, Hackenberg L, Niemeyer T, Halm H. Comparative analysis of pedicle screw and hook instrumentation in posterior correction and fusion of idiopathic thoracic scoliosis. Eur Spine J 2002;11:336-43.

12. Yilmaz G, Borkhuu B, Dhawale AA, et al. Comparative analysis of hook, hybrid, and pedicle screw instrumentation in the posterior treatment of adolescent idiopathic scoliosis. J Pediatr Orthop 2012;32:490-9.

13. Vallespir GP, Flores JB, Trigueros IS, et al. Vertebral coplanar alignment: a standardized technique for three dimensional correction in scoliosis surgery: technical description and preliminary results in Lenke type 1 curves. Spine (Phila Pa 1976) 2008;33:158897.

14. Chang KW. Cantilever bending technique for treatment of large and rigid scoliosis. Spine (Phila $\mathrm{Pa}$ 1976) 2003;28:2452-8.

15. Kuklo TR, Potter BK, Lenke LG. Vertebral rotation and thoracic torsion in adolescent idiopathic scoliosis: what is the best radiographic correlate? J Spinal Disord Tech 2005;18:139-47. 